TITLE:

\title{
Energy states in ZnSe-GaAs heterovalent quantum structures
}

AUTHOR(S):

Funato, M; Fujita, S; Fujita, S

CITATION:

Funato, M ...[et al]. Energy states in ZnSe-GaAs heterovalent quantum structures. PHYSICAL REVIEW B 1999, 60(24): 16652-16659

ISSUE DATE:

1999-12-15

URL:

http://hdl.handle.net/2433/39835

RIGHT:

Copyright 1999 American Physical Society 


\title{
Energy states in ZnSe-GaAs heterovalent quantum structures
}

\author{
Mitsuru Funato, Shizuo Fujita, and Shigeo Fujita \\ Department of Electronic Science and Engineering, Kyoto University, Kyoto 606-8501, Japan
}

(Received 13 April 1999; revised manuscript received 3 August 1999)

\begin{abstract}
We investigate the energy states in ZnSe-GaAs heterovalent quantum structures. In the ZnSe-GaAs heterovalent heterostructures, the band offsets are controllable, and therefore different offsets can artificially be put at both sides of the GaAs wells through the control of the growth sequence forming the interfaces, which brings unique characteristics on the heterovalent quantum structures. The characteristic features are examined by the optical-absorption measurement. Dependence of the absorption edge on the offsets and the well width reveals the presence of an electric field; by changing the valence-band offset at one side of the GaAs well from 0.6 to $1.1 \mathrm{eV}$, while keeping that at the other side constant at $0.6 \mathrm{eV}$, we observe that the absorption edge energies are shifted toward lower energy and that the degree of the redshift depends on the GaAs well width. These experimental results are well explained by the theoretical calculation of the Poisson and Schrödinger equations. On the other hand, the barrier width dependence exhibits the anomalous behavior that cannot be expressed by the theoretical analyses and is interpreted in terms of the charge transfer within the barrier region. [S0163-1829(99)15047-1]
\end{abstract}

\section{INTRODUCTION}

Heterovalent heterostructures such as ZnSe-GaAs and GaAs-Ge possess an attractive characteristic that band offsets between the constituent materials are variable. Different from isovalent heterostructures, the heterovalent heterostructures include interface electronic dipoles owing to inevitably formed donor and acceptor bonds, which play a crucial role in determining the band offsets. The strength and direction of the dipoles depend sensitively on the microscopic interface atomic configuration and, thus, so do the band offsets. Variations of the band offsets for $\mathrm{ZnSe}-\mathrm{GaAs}, \mathrm{Ge}-\mathrm{GaAs}$, and Si$\mathrm{GaP}$ have been theoretically predicted to be as large as $1.0,{ }^{1,2}$ $0.6,{ }^{3,4}$ and $0.8 \mathrm{eV},{ }^{3}$ respectively.

Experimentally, the effect of the interfacial properties on the tunability of the band offsets has already been investigated for several prototypical lattice matched and theoretically well-understood heterostructures. ${ }^{1,5-8} \mathrm{ZnSe}-\mathrm{GaAs}$ is one of the most suitable material combinations for the study of heterovalency because the lattice-mismatch is as small as $0.27 \%$ at room temperature (RT) and modification of the properties induced by the lattice mismatch is not significant. We have recently demonstrated the controllability of the band offsets in ZnSe-on-GaAs(001) [ZnSe/GaAs(001)] (Refs. 5 and 9) and GaAs-on-ZnSe(001) [GaAs/ZnSe(001)] (Ref. 6) heterostructures; the valence-band offsets could be tuned from 0.6 to $1.1 \mathrm{eV}$ by manipulating the interfacial atomic geometry through precise control of the flow sequence of the source precursors at the interfaces. These studies opened a way to fabricate various multilayered structures from the $\mathrm{ZnSe}-\mathrm{GaAs}$ system. For example, the offset divided into both the valence and the conduction bands may lead to formation of quantum structures, while, if the offset is put mainly on either the valence or the conduction band, double heterojunction bipolar or phototransistors with the high injection efficiency can be realized.

In this study, we fabricate the $\mathrm{ZnSe}-\mathrm{GaAs}$ heterovalent quantum structures in which the band offsets on both sides of the GaAs wells are independently designed and characterize their properties chiefly originating from heterovalency. The fabricated structures are mostly multiple quantum wells (MQW's) and partially superlattices. When the band offsets at both interfaces are different, the quantum structures should contain an electric field. Since the intensity of the electric field is changeable via control of the band offsets, the properties of the quantum structures are variable as well. So far, the effects of electric fields on the nature of the eigenstates have been investigated using the conventional isovalent quantum structures such as a $\mathrm{GaAs}-\mathrm{Al}_{x} \mathrm{Ga}_{1-x} \mathrm{As}$ system. If each well in a quantum structure is sufficiently isolated, which is the situation in a QW structure, the energy gaps will be shifted toward lower energy by the electric field. ${ }^{10}$ In the case of superlattices, the electric field causes Wannier-Stark localization. ${ }^{11}$ In these studies, however, the electric field was applied externally by, for example, pin and nin structures. On the other hand, a characteristic of the heterovalent quantum structures is that a new parameter of the band offset for designing the electronic properties enables the electric field to be included internally. Furthermore, since the tunability of the offsets depends on the interfacial dipoles being a function of the interfacial charge distribution, the structures that rearrange the charge distribution may alter the electronic properties. In this paper, we investigate dependence of the energy states in the $\mathrm{ZnSe-GaAs}$ heterovalent quantum structures, which include the intrinsic electric field, on the offsets, the well width, and the barrier width to provide an insight into their basic properties. The experimental results obtained by optical-absorption measurement at $22 \mathrm{~K}$ are compared with the results of the theoretical analyses using the Poisson and Schrödinger equations.

\section{THEORETICAL BACKGROUNDS}

The electronic properties of a quantum structure with an electric field are described by the one-dimensional Poisson and Schrödinger equations. Strictly, these equations should 
(a)

(b)

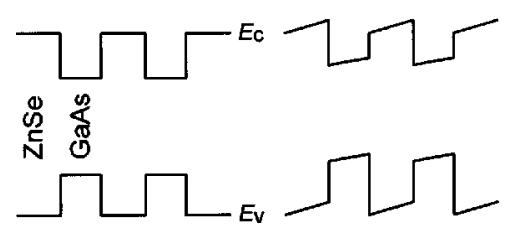

(c)

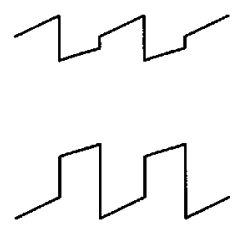

FIG. 1. Calculated potential profiles of the ZnSe-GaAs heterovalent MQW's with different pairs of the valence-band offsets at both sides of GaAs: (a) 0.6/0.6 eV, (b) 0.6/0.9 eV, and (c) 0.6/1.1 $\mathrm{eV}$. The $\mathrm{ZnSe}$ and GaAs layers are 110- and 100- $\AA$ thick, respectively. $E_{c}$ and $E_{\mathrm{v}}$ indicate the conduction and the valence band, respectively.

be solved self-consistently; though for simplicity, the following approximation has been made in this study. The quantum confinement effect was not considered in the Poisson equation, and the carrier concentrations were written in classical formulations using the Ehrenberg approximation. ${ }^{12}$ Within the limits of this classical approximation, the potentialenergy profiles are uniquely determined only by the Poisson equation. Therefore, eigenstates are, then, calculated by solving the Schrödinger equation, where carriers experience the potential profile derived from the Poisson equation. Although this procedure is not self-consistent and may degrade the quantitative accuracy, the included error does not seem to be serious due to the following reasons. If the well width is thick, the classical density of states well approximates that with the quantum effect. The thin well, on the other hand, induces the substantial difference in the density of states, although the electric field, in turn, does not influence eigenstates remarkably. Therefore, the electronic properties of the heterovalent quantum structures can be outlined by the procedure adopted here with relative easiness.

\section{A. Potential-energy profiles}

Since the samples fabricated in this study are multilayered quantum structures, the Poisson equation is solved with the periodic boundary conditions. Figure 1 shows some of the calculated potential profiles of the MQW's. The dimensions of the GaAs wells and the ZnSe barriers are 100 and 110 $\AA$, respectively. The pairs of the valence-band offsets at both sides of the GaAs wells are (a) 0.6/0.6, (b) 0.6/0.9, and (c) $0.6 / 1.1 \mathrm{eV}$. These structural and physical parameters were chosen, referring to the actually fabricated structures (cf. Sec. III). Here, $\mathrm{ZnSe}$ is assumed to be $n$ type with donor concentration of $1 \times 10^{11} \mathrm{~cm}^{-3}$, while GaAs is $p$ type with $5 \times 10^{17} \mathrm{~cm}^{-3}$. These quantities are the experimental results obtained from Hall-effect measurements of thick layers but are not essential as will be shown later. It is obvious from Fig. 1 that the electric field, which corresponds to the inclination of the potential profile, becomes stronger by the increase of the difference of the valence-band offsets and that its intensity is approximately uniform in either the $\mathrm{ZnSe}$ or the GaAs region.

Based on Fig. 1, the average electric fields in the well and the barrier regions were estimated on the assumption that the electric field was uniform in each region. The results are shown in Fig. 2, where the valence-band offset at one side of GaAs is $0.6 \mathrm{eV}$ and that at the other side is a variable des-

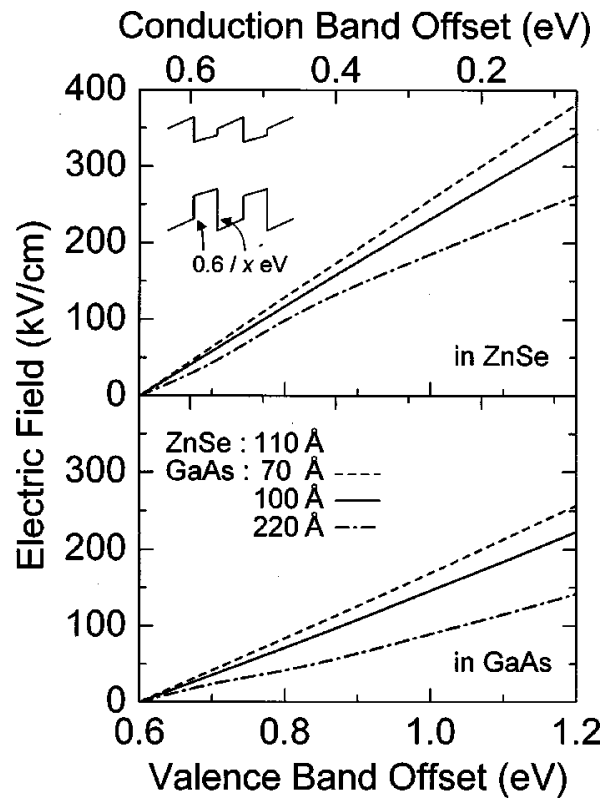

FIG. 2. Estimated electric fields in $\mathrm{ZnSe}$ and GaAs. The dimensions of $\mathrm{ZnSe}$ and GaAs are 110 and $100 \AA$, respectively. The valence-band offset at one side of GaAs is $0.6 \mathrm{eV}$ and that at the other side is a variable shown at the horizontal axis.

ignated at the horizontal axis. Variation due to the well width is also compared. The intensities of the electric fields are almost proportional to the difference of the valence-band offsets and are stronger in the MQW's with the narrower wells.

As is well known, impurity concentration often influences the potential profile of semiconductor heterojunctions. Surprisingly, however, it was found that the potential profiles of the heterovalent quantum structures hardly depended on the impurity concentration and that those were mainly determined by the difference of the valence-band offsets at both sides of the GaAs wells. For example, when the valenceband offsets at both interfaces are 0.6 and $1.1 \mathrm{eV}$, difference of the acceptor concentrations in GaAs of $1 \times 10^{14}$ and 5 $\times 10^{17} \mathrm{~cm}^{-3}$ resulted in only a few percent of variation of the electric fields, that is, $195 \mathrm{kV} / \mathrm{cm}$ and $184 \mathrm{kV} / \mathrm{cm}$ in GaAs, respectively. Furthermore, $p$ - and $n$-GaAs with the same impurity concentration of $5 \times 10^{17} \mathrm{~cm}^{-3}$ exhibited the almost identical potential profiles; electric fields in GaAs were $184 \mathrm{kV} / \mathrm{cm}$ for $p$-GaAs, while $183 \mathrm{kV} / \mathrm{cm}$ for $n$-GaAs. The similar tendency was observed by changing the impurity concentration in $\mathrm{ZnSe}$. The reason for this independence on the impurity concentration is that the potential inclination due to the difference of the valence-band offsets is remarkably greater than that induced by donor and acceptor impurities and determines the potential profiles predominantly.

\section{B. Energy eigenstates}

Under the presence of the electric field revealed by the Poisson equation, the Schrödinger equation was solved by the variational method ${ }^{13}$ to estimate the energy eigenstates. A single QW with $\mathrm{ZnSe}$ barriers of infinite width was used as a model for the calculation. This approximation is valid for most of the present quantum structures, because their barrier width is typically about $110 \AA$ and the penetration of the calculated wave function into the $\mathrm{ZnSe}$ barrier regions 


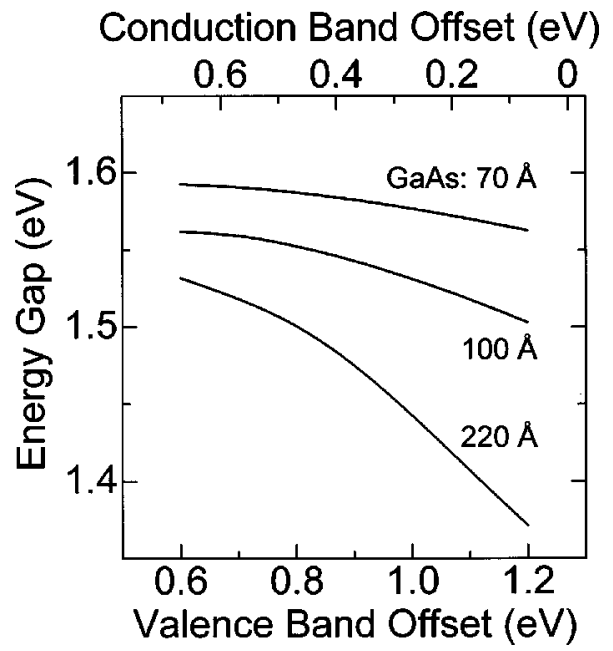

FIG. 3. Calculated energy gaps as a function of the valenceband offset. The dimension of the GaAs well is a parameter and that of the $\mathrm{ZnSe}$ barrier used for the calculation of the potential profiles is $110 \AA$.

was totally within about $100 \AA$. Namely, these are MQW's, in which the wave function in each well is thoroughly isolated and hardly interacts with one another. The used variational wave functions are basically the same with those in Ref. 13, though were modified to satisfy the boundary conditions of $\psi_{\mathrm{GaAs}}=\psi_{\mathrm{ZnSe}}$ and $m_{\mathrm{GaAs}}^{*}{ }^{-1}\left(d \psi_{\mathrm{GaAs}} / d z\right)$ $=m_{\mathrm{ZnSe}}^{*}{ }^{-1}\left(d \psi_{\mathrm{ZnSe}} / d z\right)$ at the interfaces, where $\psi$ and $m^{*}$ denote the wave functions and the effective masses for the indicated material. The energy gap was estimated from the eigenstates determined by the Schrödinger equation, assuming the energy band gap of GaAs at $20 \mathrm{~K}$ as $1.521 \mathrm{eV}$.

Examples of the calculated energy gaps are shown in Fig. 3 , where the dimension of the GaAs well is a parameter and that of the $\mathrm{ZnSe}$ barrier used for the calculation of the potential profiles is $110 \AA$. Here, the valence-band offset was $0.6 \mathrm{eV}$ for one side of GaAs and that for another side was taken as a variable, as is the same with Fig. 2. It is clearly seen that the larger valence-band offset, which induces the stronger electric field (Fig. 2), causes the redshift of the energy gap and that its degree depends on the well width. If the pair of the valence-band offsets is the same, the wide well weakens the electric field in GaAs as shown in Fig. 2. However, the redshift in Fig. 3 is more striking with the wider well. Therefore, it can be concluded that the effect of the electric field appears more explicitly in the quantum structure with the wider well. This is well explained in terms of a spatial shift of the electron and the hole wave functions induced by the electric field. The wave functions after the spatial shift, which occurs more easily in the wider wells due to the weak quantum confinement effect, experience the potential valleys and lower the energy states.

In this study, the periodic potential profile like Fig. 1 is considered as a first trial. However, actually, the band offset at each interface in a multilayered structure can be designed independently, and besides, the well and barrier widths that influence the intensity of the electric fields are variable as well. Therefore, various potential profiles can be realized. In this sense, it is crucially important to give a guiding principle for designing the electronic properties of the heterovalent quantum structure. Here, we identify which is most responsible for the redshift, the electric field in the GaAs wells $\left(F_{w}\right)$, that in the ZnSe barriers $\left(F_{b}\right)$, or the band offsets, by examining the contribution of each factor to the energy gap separately. The potential profiles for this calculation were determined irrespectively of the result of the Poisson equation, and the GaAs well width was kept constant at $100 \AA$.

It was found that the energy gap shifted from 1.561 to $1.362 \mathrm{eV}$ by the presence of $F_{w}$ from 0 to $500 \mathrm{kV} / \mathrm{cm}$, respectively, where $F_{b}$ was zero and the pair of the valenceband offsets was $0.6 / 1.1 \mathrm{eV}$. The sign of positive in $F_{w}$ means that its vector is pointing toward the interface with the valence-band offset of $1.1 \mathrm{eV}$. The redshift was also observed by $F_{w}$ in the opposite direction, that is, $F_{w}$ from 0 to $-500 \mathrm{kV} / \mathrm{cm}$ brought the energy gaps from 1.559 to 1.287 $\mathrm{eV}$, respectively. Under the negative $F_{w}$, both electron and hole wave functions are pushed to the interfaces with the smaller band offsets and penetration into the barrier regions becomes pronounced, compared with that under the positive $F_{w}$. This causes the quantitative difference of the energy gaps of 1.362 and $1.287 \mathrm{eV}$ under $\pm 500 \mathrm{kV} / \mathrm{cm}$. However, an important result of this calculation is that $F_{w}$ can potentially bring about the variation of the energy gap in hundreds meV.

On the other hand, $F_{b}$ in the range from -500 to 500 $\mathrm{kV} / \mathrm{cm}$ with zero $F_{w}$ and the pair of the valence-band offsets of 0.6/1.1 eV resulted in a trivial change of the energy gap in the order of sub meV. Although this variation due to $F_{b}$ is enlarged by the increase of $F_{w}$, its order is still meV even with $F_{w}=500 \mathrm{kV} / \mathrm{cm}$. The reason for the enhancement of the redshift by greater $F_{w}$ is that the wave functions are spatially shifted toward the well barriers by $F_{w}$ to be more sensitive to the electric field in the barriers, i.e., $F_{b}$.

As for the effect of the band offset on the energy gap, the calculation was carried out, keeping the valence-band offset at one side of the GaAs well at $0.6 \mathrm{eV}$ and changing that at the other side between 0.6 and $1.1 \mathrm{eV} . F_{b}$ was assumed to be $0 \mathrm{~V} / \mathrm{cm}$. The degree of the energy shift owing to control of the band offsets depends on $F_{w}$. The larger $F_{w}$ causes the greater energy shift, although even under $F_{w}$ of $500 \mathrm{kV} / \mathrm{cm}$, the shift due to the change of the valence-band offsets from 0.6 to $1.1 \mathrm{eV}$ was estimated to be only about $15 \mathrm{meV}$. From the above discussions, it can be concluded that the offset itself is not important and that the electric field in the GaAs wells as a function of the offset plays a principal role in determining the energy gap.

In Secs. II A and II B, the existence of strain, which modifies the energy bands, has been neglected. Here, the validity of this assumption is proved. Since the $\mathrm{ZnSe}-\mathrm{GaAs}$ quantum heterostructures are grown on the GaAs substrates in this study, only the ZnSe layers are strained to achieve pseudomorphic growth as verified in Sec. III B. The lattice parameter of $\mathrm{ZnSe}$ is $5.6686 \AA$, while that of GaAs is $5.6533 \AA$ at $\mathrm{RT}$, and thus the strain in $\mathrm{ZnSe}$ is compressive. The effect of the compressive strain on the energy band of $\mathrm{ZnSe}$ is estimated with the model-solid theory proposed by Van de Walle et al. ${ }^{14,15}$ The used physical parameters are the same with those in Ref. 15. As a result, it was found that the compressive strain in $\mathrm{ZnSe}$ makes the band offsets larger by $2 \mathrm{meV}$ for heavy holes, by $10 \mathrm{meV}$ for light holes, and by 9 $\mathrm{meV}$ for electrons. Using these values, the Poisson equation 


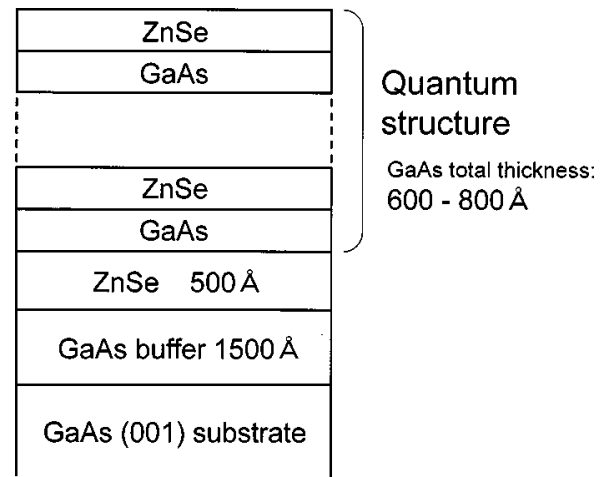

FIG. 4. Schematic diagram of the fabricated $\mathrm{ZnSe-GaAs}$ MQW's.

was solved again. However, there was no difference in the electrostatic potentials with and without strain. In other words, the change provided in the potential-energy profiles by the strain was only band offsets, and the electric fields in $\mathrm{ZnSe}$ and GaAs remain unchanged. This is quite reasonable because, as was mentioned in the previous section, the potential profile is governed chiefly by the difference of the valence-band offsets at both interfaces, and the modification of the energy band owing to the compressive strain does not alter the difference of the offsets. Then, the Schrödinger equation was solved using the newly calculated potentialenergy profile. As is easily deduced, since the electric field in GaAs, which was revealed to be essential to determine energy eigenvalues, is unchanged by the strain, the energy shift seems to be considerably small, and indeed, it was estimated for MQW with $100 \AA \mathrm{GaAs}$ to be in the order of meV or less. Compared with the variation of the energy gap shown in Fig. 3, this quantity is negligible, which validates the assumption made for the present calculation. We will also neglect the influence of strain on the experimental data.

\section{EXPERIMENTAL RESULTS AND DISSCUSION}

\section{A. Sample preparation}

The $\mathrm{ZnSe-GaAs} \mathrm{quantum} \mathrm{structures} \mathrm{were} \mathrm{fabricated} \mathrm{on}$ $\mathrm{GaAs}(001)$ substrates by metal-organic vapor phase epitaxy (MOVPE). The source precursors for GaAs and $\mathrm{ZnSe}$ were triethylgallium (TEGa) and tertiarybutylarsine (TBAs), and diethylzinc (DEZn) and dimethylselenium (DMSe), respectively. The sample structure is schematically shown in Fig. 4. Growth of the samples was initiated by a GaAs buffer layer $1500-\AA$ thick at $700^{\circ} \mathrm{C}$. To obtain an atomically flat surface, post-growth annealing was conducted at $700{ }^{\circ} \mathrm{C}$ for $10 \mathrm{~min}$. Next a 500 - $\AA$-thick $\mathrm{ZnSe}$ region was grown at $450^{\circ} \mathrm{C}$ primarily to act as an etch stop layer for substrate removal. Finally, a ZnSe-GaAs quantum structure was grown at $450{ }^{\circ} \mathrm{C}$. The period of the quantum structure was designed so as to make the total thickness of the GaAs wells 600-800 $\AA$. The detailed growth conditions are found in Refs. 5 and 6, and references therein. The valence-band offsets in both $\mathrm{ZnSe} / \mathrm{GaAs}$ and $\mathrm{GaAs} / \mathrm{ZnSe}$ heterostructures are controllable. In this study, however, to simplify the discussion, the former was kept constant at $0.6 \mathrm{eV}$ for all samples, while the latter was changed between 0.6 and $1.1 \mathrm{eV}$. For this control of the band offset, the interval duration between the $\mathrm{ZnSe}$

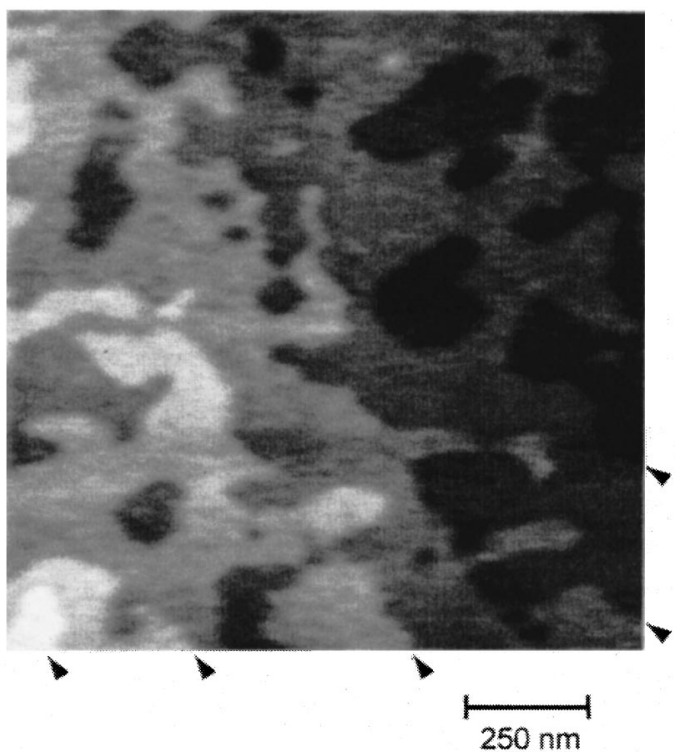

FIG. 5. Typical AFM image of the surface of the ZnSe-GaAs quantum structures. Arrowheads indicate 1 monomolecular steps (2.8 $\AA$ in height).

and GaAs growth, during which only hydrogen was supplied for purging the $\mathrm{ZnSe}$ source precursors, was set at $5 \mathrm{~s}$, and the GaAs growth was initiated after the exposure of $\mathrm{ZnSe}$ to TBAs for 2-20 s (see Ref. 6 for more details). The influence of this artificial control of the band offsets on the electronic states is assessed by optical-absorption measurement at $22 \mathrm{~K}$.

\section{B. Structural properties}

In order to extract differences of the optical properties due to the interface engineering, it is important to clarify the structural properties, because the structural properties may also contribute to the modification of the optical properties of the quantum structures. For this purpose, the surface morphology and the crystallinity were investigated by means of atomic force microscopy (AFM) and $\mathrm{x}$-ray diffraction (XRD), respectively.

The AFM observation was carried out in air immediately after the growth. There is no evident difference associated with the interface control, and the surfaces for all samples exhibited atomically flat terraces and monomolecular steps (2.8 $\AA$ in height) as shown in Fig. 5. This result suggests that, regardless of the manner to prepare the interfaces, the growths of both $\mathrm{ZnSe} / \mathrm{GaAs}$ and $\mathrm{GaAs} / \mathrm{ZnSe}$ are in the twodimensional growth mode under the conditions employed here and that the interfaces are abrupt.

The XRD patterns were taken in the vicinity of the GaAs 004 diffraction, using $\mathrm{Cu} K \alpha_{1}(1.5405 \AA)$ radiation as an $\mathrm{X}$-ray source. Figure 6 shows an example of the XRD profiles, in which both experimental and simulated profiles are illustrated. The sample, in this particular case, is the 8 periods MQW with the expected dimensions of $110 \AA$ for $\mathrm{ZnSe}$ and $100 \AA$ for GaAs. Comparison between the experimental result and the simulation based on the dynamical diffraction theory indicates that those are 107 and $99 \AA$, which are very close to the designed parameters. In the experimental profile, in addition to a fundamental peak $(n=0)$, well-defined satellite peaks labeled $n= \pm 1$ are clearly detected. The peak 


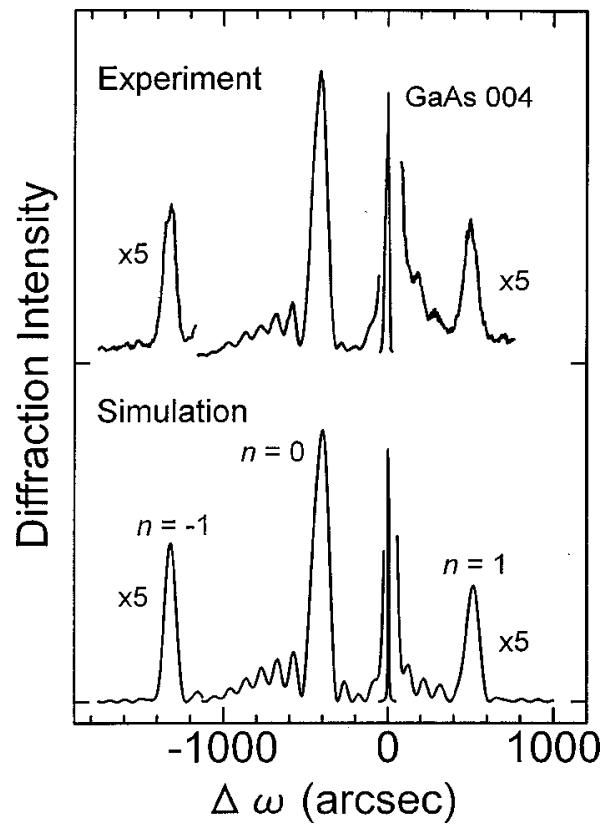

FIG. 6. An example of the XRD profiles of the ZnSe-GaAs quantum structures. The agreement between the experimental result and the theoretically calculated profile indicates the high degree of the structural perfectness.

intensity ratios between the satellite and the fundamental peaks and the full width at the half maximum (FWHM) agree quite well with those derived from the dynamical simulation, indicating the high crystalline quality, high periodicity, and high interface abruptness in the ZnSe-GaAs quantum structure. The $\mathrm{ZnSe}$ etch stop layer $500-\AA$ thick contributes to the increase of the diffraction intensity between -1000 and -500 arcsec, for which the experiment again does agree with the calculation. In the simulation, $\mathrm{ZnSe}$ was assumed to be deformed so as to maintain coherent growth, and the correspondence with the experimental result supports this assumption. The entire region of the quantum structure is grown coherently on the GaAs substrate with few dislocations. It should be emphasized that fairly good agreement between experiment and simulation was confirmed for all samples, which allows us to interpret the optical properties without caring about structural imperfections.

\section{Optical-absorption properties}

We will move to the discussion on the optical-absorption measurement. For the absorption measurement, the substrate of the samples was removed by a selective $\mathrm{NaOH}(1 \mathrm{~N})$ : $\mathrm{H}_{2} \mathrm{O}_{2}=1: 1$ (in volume) etching solution at RT. The light from a halogen lamp was irradiated to the samples, and the transmitted light was detected using a cooled charge coupled device (CCD) and a 50-cm monochromator. The intensity of the irradiated light was made as weak as possible so as to avoid unexpected modification of the band alignment due to the photogenerated carriers. Under the present experimental setup, no luminescence was observed, and therefore, the detected signals reflect only the absorption properties.

Figure 7 shows a typical $22 \mathrm{~K}$ absorption spectrum of the $\mathrm{ZnSe}-\mathrm{GaAs}$ heterovalent quantum structure, which consists

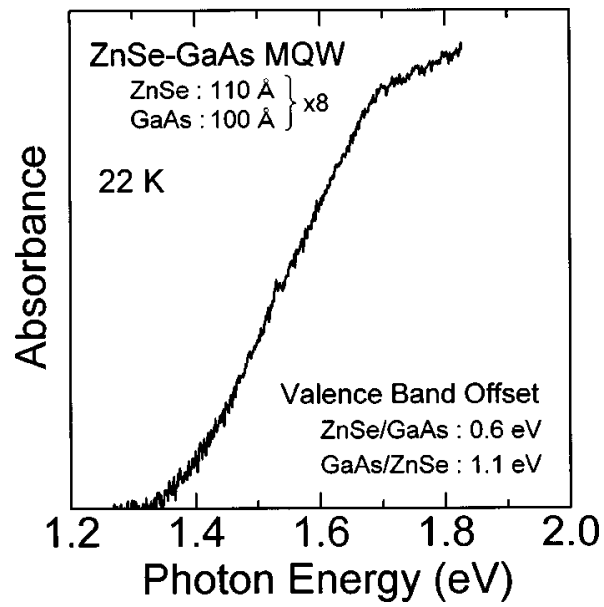

FIG. 7. Typical absorption spectrum of the ZnSe-GaAs heterovalent quantum structure measured at $22 \mathrm{~K}$. The sample is the eight periods MQW consisting of $110 \AA \mathrm{ZnSe}$ and $100 \AA \mathrm{GaAs}$. The valence-band offsets at $\mathrm{ZnSe} / \mathrm{GaAs}$ and $\mathrm{GaAs} / \mathrm{ZnSe}$ are designed to be 0.6 and $1.1 \mathrm{eV}$, respectively.

of eight periods of $110 \AA \mathrm{ZnSe}$ and $100 \AA$ GaAs. The valence-band offsets at $\mathrm{ZnSe} / \mathrm{GaAs}$ and $\mathrm{GaAs} / \mathrm{ZnSe}$ are 0.6 and $1.1 \mathrm{eV}$, respectively. Since excitonic absorption peaks were not detected, the following discussion has been done based on the absorption edge, which was defined as the energy determined by extrapolation of linear dependence of the square of the absorbance on irradiated photon energy into zero absorbance. It must be noted that the absorption edge energy $\left(E_{\text {abs }}\right)$ is generally lower than the energy gap $\left(E_{g}\right)$ due to absorption by impurities and/or excitons, that is,

$$
E_{\mathrm{abs}}=E_{g}-\Delta E_{\mathrm{abs}},
$$

where $\Delta E_{\text {abs }}$ indicates the variation caused by the impurity and/or exciton absorption. In this study, however, the contri-

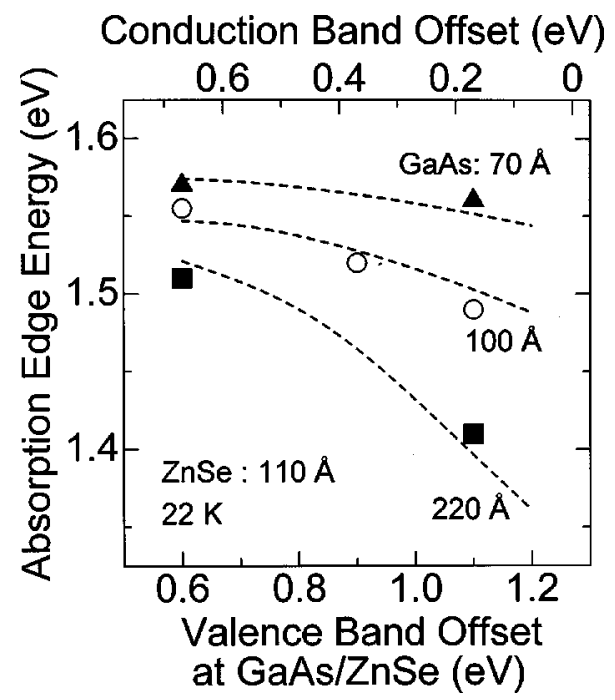

FIG. 8. The absorption edge energies of the MQW's with different pairs of the valence-band offsets at both sides of the GaAs wells. The valence band offset at the $\mathrm{ZnSe} / \mathrm{GaAs}$ interfaces is constant at $0.6 \mathrm{eV}$. Thickness of $\mathrm{ZnSe}$ is $110 \AA$ for all samples in this figure. The broken lines indicate the theoretically determined absorption energies $\left(E_{\mathrm{abs}}\right)$, which were evaluated by subtracting the exciton binding energies from the energy gaps $\left(E_{g}\right)$. 
bution from impurities to $\Delta E_{\mathrm{abs}}$ is considered to be negligible. This is because the absorption edge energy of GaAs $1150-\AA$ thick grown on $\mathrm{ZnSe} / \mathrm{GaAs}$ was estimated to be $1.518 \mathrm{eV}$, and the energy difference of $3 \mathrm{meV}$ between the estimated value $(1.518 \mathrm{eV})$ and the energy band gap of GaAs at $20 \mathrm{~K}(1.521 \mathrm{eV})$ is close to the exciton binding energy of bulk GaAs ( $\sim 5 \mathrm{meV})$. In the following, $\Delta E_{\mathrm{abs}}$ is determined by referring to the exciton binding energy calculated in Ref. 16.

\section{Band offset dependence}

The absorption edge energies of the MQW's with different pairs of the valence-band offsets at both sides of the GaAs wells are compared in Fig. 8. The ZnSe barrier thickness was $110 \AA$ for the samples in Fig. 8. The valence-band offset at the $\mathrm{ZnSe} / \mathrm{GaAs}$ interface is $0.6 \mathrm{eV}$ as stated before, and that at the GaAs/ZnSe interfaces is a variable indicated at the horizontal axis. Accordingly, for example, the valenceband offset of $0.6 \mathrm{eV}$ in the figure means the flatband condition. The result with $220 \AA \mathrm{GaAs}$ and the valence-band offset of $1.1 \mathrm{eV}$ may be less reliable. Since the electron and hole wave functions are remarkably separated under the structural and physical parameters for this sample, ${ }^{17}$ the absorption is suppressed, and consequently, the signal-to-noise ratio in the experiment gets worse.

It is explicitly seen in Fig. 8 that the MQW's with the larger valence-band offset, which corresponds to the larger difference of the valence-band offsets at both sides of GaAs, exhibit the lower absorption energy and that the redshift becomes more conspicuous in the MQW's with the wider wells. The broken lines in the figure indicate the absorption energies $\left(E_{\mathrm{abs}}\right)$ calculated from Eq. (1), that is, by subtracting $\Delta E_{\text {abs }}$ from energy gaps $\left(E_{g}\right)$ shown in Fig. 3. As $\Delta E_{\text {abs }}$ in Eq. (1), which is equal to the exciton binding energy, 19, 15 , and $11 \mathrm{meV}$ were used for MQW's with the well width of 70,100 , and $220 \AA$, respectively, according to the calculated results in Ref. 16. It is worth noting that those exciton binding energies are larger than those in the GaAs-AlAs system owing to the large difference in the dielectric constants between GaAs and ZnSe. Strictly, the exciton binding energy depends on the intensity of the electric field; though, here, it was assumed to be constant at the value without electric fields as a first trial. The reasonable agreement between the experimental results and the theoretical calculations indicates that the electronic properties in the heterovalent MQW's are well described by the potential profiles shown in Fig. 1 and by the energy eigenvalues determined by the profiles. Moreover, it can be concluded that the modification of the exciton binding energy due to the presence of the electric field is within the present experimental resolution. It is interesting to note that the results obtained here prove the capability of controlling the electronic properties of heterovalent MQW's through the control of the band offsets, which differs considerably from isovalent systems.

\section{Well width dependence}

The quantum confinement effect was examined by the well width dependence of the absorption edges. The samples used for this experiment possess the ZnSe barriers $110-\AA$ thick. Two series of the samples, that is, with and without

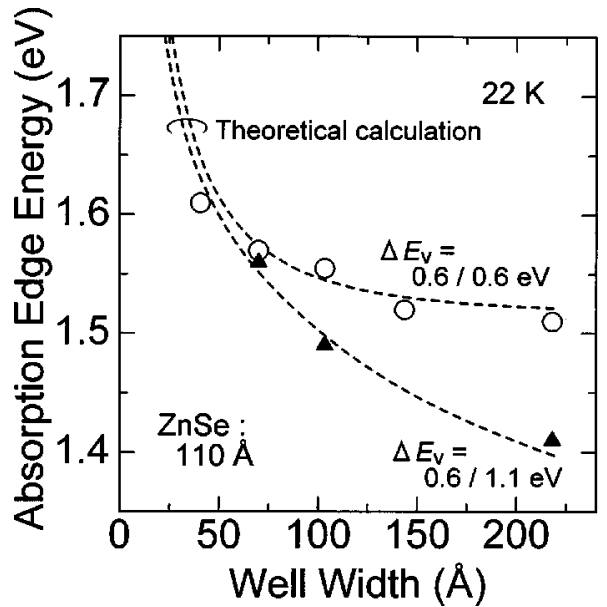

FIG. 9. Well width dependence of the absorption edges. The ZnSe barriers are 110- $\AA$ thick. The pair of the valence-band offsets $\left(\Delta E_{\mathrm{v}}\right)$ at both sides of GaAs is used as a parameter. The broken lines are the theoretically calculated absorption energies with the consideration of the exciton binding energies.

difference between the band offsets at both sides of GaAs, were investigated. For the former, the valence-band offsets at the $\mathrm{ZnSe} / \mathrm{GaAs}$ and the $\mathrm{GaAs} / \mathrm{ZnSe}$ interfaces are 0.6 and 1.1 $\mathrm{eV}$, respectively, which yields the internal electric field. In the latter case, both valence-band offsets are $0.6 \mathrm{eV}$, and thus, there is no electric field.

The results are shown in Fig. 9. The broken lines are the theoretically calculated absorption energies with the consideration of the exciton binding energy. Similar to Fig. 8, the fairly good agreement between the experiment and the calculation was again attained, indicating the achievement of the quantum confinement. Furthermore, it is clearly observed from either the experiment or the calculation that as the well width is widened, the variation of the energy gap due to the electric field becomes pronounced, as was already pointed out in Sec. II B. The results here support the conclusion in the previous section that the electronic properties of heterovalent MQW's are controllable by the interface control.

\section{Barrier width dependence}

Finally, we describe the barrier width dependence. As decreasing the barrier width, quantum structures are generally converted from MQW's to superlattices in which the wave functions in wells interact with each other to form minibands. In the conventional isovalent quantum structures, formation of the minibands due to the conversion from MQW's to superlattices appears in the absorption properties as the lowered absorption edge. Our interest is what is observed in the heterovalent quantum structures by changing the barrier width. In order to elicit the difference between the isovalent and heterovalent quantum structures, different valence-band offsets were set at the $\mathrm{ZnSe} / \mathrm{GaAs}$ and the $\mathrm{GaAs} / \mathrm{ZnSe}$ interfaces as 0.6 and $1.1 \mathrm{eV}$, respectively. The dimension of the GaAs wells was $100 \AA$.

Before showing the experimental details, the theoretical analyses are briefly performed. First, the miniband structures were calculated by the Kronig-Penny model assuming the quantum structures without electric fields. It was demonstrated that the first miniband for electrons was formed with 


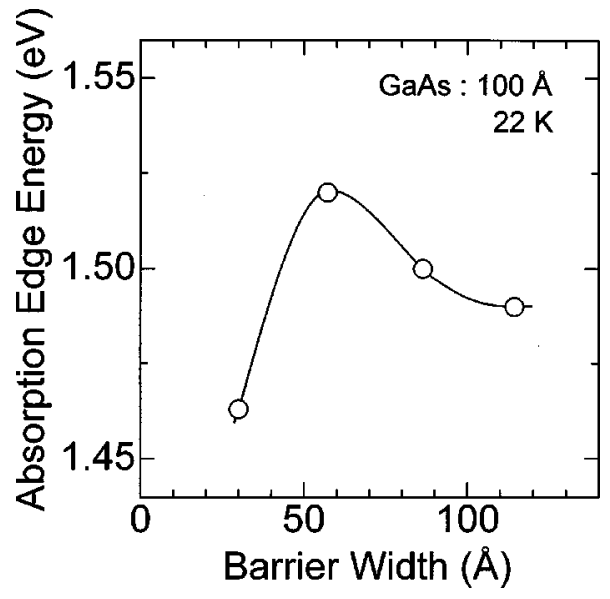

FIG. 10. Barrier width dependence of the absorption edges. The dimension of GaAs is $100 \AA$. The originally designed valence-band offsets at $\mathrm{ZnSe} / \mathrm{GaAs}$ and $\mathrm{GaAs} / \mathrm{ZnSe}$ are 0.6 and $1.1 \mathrm{eV}$, respectively.

the barriers less than $70-\AA$ thick and that its width at the barrier width of $30 \AA$ was $1.5 \mathrm{meV}$. Since the presence of an electric field localizes the carriers and prevents the formation of the minibands, the result above suggests that the calculation process of the Schrödinger equation employed in this study does not involve a serious error for the multiple quantum structures with barriers thicker than $70 \AA$. For superlattices with barriers thinner than $70 \AA$, the quantitative accuracy is surely degraded, although it is still possible to extract the trend of the energy states against the barrier width by the same technique. This is because the variation of the energy levels due to the conversion into superlattices, that is, due to the formation of the minibands, is relatively small as was estimated to be $1.5 \mathrm{meV}$ for the barriers $30-\AA$ A thick. Consequently, it was found that as the barrier width was reduced from 110 to $30 \AA$, the electric field in the GaAs regions became stronger and the energy gap shifted monotonously from 1.518 to $1.444 \mathrm{eV}$. Namely, the theoretical prediction is that redshift will be observed also in heterovalent systems by reducing the barrier width, though that is predominantly caused by the internal electric field, differing from isovalent systems. The redshift due to formation of the minibands will be too small to be observed.

Experimentally, the absorption edge energy behaves contrary to the theoretical prediction as shown in Fig. 10. As the barrier width decreases from $110 \AA$, it is once shifted to higher energy, and then shows the redshift below $50 \AA$. Regarding the blueshift, it cannot be interpreted by the theoretical analyses so far performed in this study, and therefore, another factor must be considered. According to Figs. 2 and 3 , the blueshift means that the difference of the valence-band offsets at both sides of the GaAs wells gets smaller to weaken the electric field in GaAs. As a cause for this, we consider at present the charge transfer within the ZnSe region. Let us explain this model using Fig. 11. The tunability of the band offsets being utilized in this study is based on the formation of the electronic dipoles at the interfaces. ${ }^{5,6}$ These electronic dipoles originate from the charge transfer from donating Ga-Se bonds to accepting $\mathrm{Zn}$-As bonds across the interface. If the $\mathrm{ZnSe}$ and $\mathrm{GaAs}$ layer thicknesses are relatively thick and each interface is isolated, these dipoles are (a) Thick ZnSe

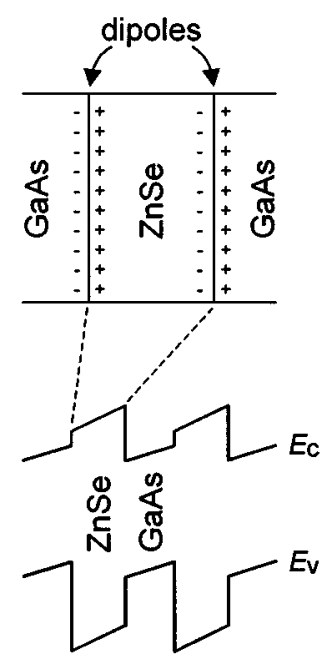

(b) Thin ZnSe

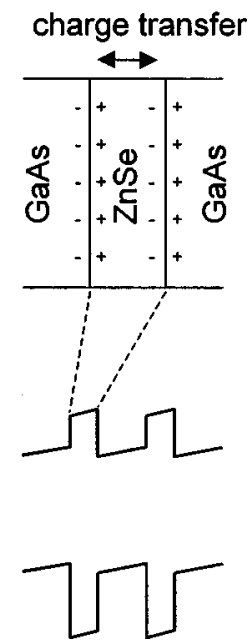

FIG. 11. A model for the blueshift observed in the barrier width dependence of the absorption edges.

preserved even after the alternating deposition of $\mathrm{ZnSe}$ and $\mathrm{GaAs}$, and the optical properties agree reasonably well with the theoretical calculation as shown in Figs. 8 and 9. This situation and the resultant potential profile are schematically illustrated in Fig. 11(a). In this figure, taking the results of Refs. 5 and 6 into account, the dipoles for the valence-band offset of $0.6 \mathrm{eV}$ are directed to $\mathrm{ZnSe}$ and those for $1.1 \mathrm{eV}$ to GaAs. Thin ZnSe, on the other hand, facilitates the interaction between interfaces and promotes the charge transfer from one side to the other side of the ZnSe barrier. As a result of this charge rearrangement within the $\mathrm{ZnSe}$ region, the dipoles at both interfaces tend to weaken each other, which makes the difference of the valence-band offsets small as shown in Fig. 11(b). Since the small difference of the offsets weakens the electric fields, the absorption energy is shifted to higher energy. This is the reason for the blueshift observed in Fig. 10.

In the above discussion with Fig. 11, the sign of the charges at both sides of $\mathrm{ZnSe}$ was opposite and the dipoles were in the same direction. However, the discussion can be extended to the dipoles with arbitrary direction. If the dipoles are in the same direction with different strength, the charge transfer occurs from the side with the higher charge density to that with the lower density, and the difference in strength of the dipoles becomes small. Therefore, similar to Fig. 11, the electric field is weaker in the quantum structures with narrower barriers, which causes the blueshift.

Concerning the redshift below $50 \AA$, the theoretical analyses stated previously in this section suggest that this is probably due to the electric field because the amount of the observed redshift is $50-60 \mathrm{meV}$, which is much greater than the calculated width of the first miniband. The reason why the effect of the electric field appears explicitly in the quantum structures with the barriers thinner than $50 \AA$ is considered as follows. The final charge distribution is decided not only by the ZnSe thickness but also by other factors such as the charges at the GaAs side of the interfaces and the donor and acceptor bonds at the interfaces. In Fig. 10, the charge transfer due to the variation of the ZnSe thickness will be completed at about $50 \AA$, and the electric field remained after 
the charge transfer is strengthened by thinning the barrier width and causes the redshift.

Although the similar charge transfer must take place in the GaAs wells by reducing the GaAs well width, observation of this phenomenon will be difficult because the influence of the electric field on the absorption energy is originally not so remarkable in the narrow wells as shown in Fig. 9. The barrier width dependence shown here is one of the unique characteristics of the heterovalent quantum structures, which is never observed with the conventional isovalent systems.

\section{CONCLUSIONS}

$\mathrm{ZnSe}-\mathrm{GaAs}(001)$ heterovalent quantum structures were fabricated, and their optical properties were assessed by the absorption measurement at $22 \mathrm{~K}$. Dependence of the absorption edge energy on the offsets, the well width, and the barrier width was investigated. The absorption edges were shifted toward lower energy by enlarging the difference of the valence-band offsets at both sides of the GaAs wells. This was an indication of the presence of the internal electric field. The well width dependence also provided evidence of the presence of the electric field. The theoretical calculation of the Poisson and Schrödinger equations well explained these experimental results. In contrast, the barrier width dependence exhibited the anomalous behavior and could not be expressed by the theoretical analyses. This unique characteristic was interpreted in terms of the charge transfer within the barrier region.

\section{ACKNOWLEDGMENTS}

This work was supported by the Ozawa and Yoshikawa Memorial Electronics Research Foundation. The authors are grateful to S. Aoki, H. Tachibana, and T. Ikuma for their collaboration in the experimental studies.
${ }^{1}$ R. Nicolini, L. Vanzetti, G. Mula, G. Bratina, L. Sorba, A. Franciosi, M. Peressi, S. Baroni, R. Resta, A. Baldereschi, J. E. Angelo, and W. W. Gerrich, Phys. Rev. Lett. 72, 294 (1994).

${ }^{2}$ A. Kley and J. Neugebauer, Phys. Rev. B 50, 8616 (1994).

${ }^{3}$ R. G. Dandrea, S. Froyen, and A. Zunger, Phys. Rev. B 42, 3213 (1990).

${ }^{4}$ K. Kunc and R. M. Martin, Phys. Rev. B 24, 3445 (1981).

${ }^{5}$ M. Funato, S. Aoki, Sz. Fujita, and Sg. Fujita, J. Appl. Phys. 82, 2984 (1997).

${ }^{6}$ M. Funato, S. Aoki, Sz. Fujita, and Sg. Fujita, J. Appl. Phys. 85, 1514 (1999).

${ }^{7}$ R. W. Grant, J. R. Waldrop, S. P. Kowalczyk, and E. A. Kraut, J. Vac. Sci. Technol. B 3, 1295 (1985).

${ }^{8}$ M. Dahmen, U. Rau, M. Kawanaka, J. Sone, and J. H. Werner, Appl. Phys. Lett. 62, 261 (1993).

${ }^{9}$ In this paper, a (material $A$ )-on-(material $\left.B\right)$ structure is denoted as $A / B$ and a multilayered structure consisting of (material $A$ ) and (material $B)$ as $A-B$.

${ }^{10}$ D. A. B. Miller, D. S. Chemla, T. C. Damen, A. C. Gossard, W. Wiegmann, T. H. Wood, and C. A. Burrus, Phys. Rev. B 32, 1043 (1985)

${ }^{11}$ E. E. Mendez, F. Agulló-Rueda, and J. M. Hong, Phys. Rev. Lett. 60, 2426 (1988).

${ }^{12}$ W. Ehrenberg, Proc. Phys. Soc., London, Sect. A 63, 75 (1950).

${ }^{13}$ G. Bastard, E. E. Mendez, L. L. Chang, and L. Esaki, Phys. Rev. B 28, 3241 (1983).

${ }^{14}$ C. G. Van de Walle and R. M. Martin, Phys. Rev. B 34, 5621 (1986).

${ }^{15}$ K. Shahzad, D. J. Olego, and C. G. Van de Walle, Phys. Rev. B 38, 1417 (1988).

${ }^{16}$ M. Kumangai and T. Takagahara, Phys. Rev. B 40, 12359 (1989).

${ }^{17}$ The overlap integral between electron and hole wave functions was calculated to be about $10 \%$ of that without electric field. 